\title{
Rheumatoid Arthritis Does Not Negatively Impact Outcomes of Patients Admitted for Atrial Fibrillation
}

Ehizogie Edigin ${ }^{1}$, Emmanuel Akuna ${ }^{1}$, Iriagbonse Asemota ${ }^{1}$, Precious Eseaton ${ }^{2}$, Pius E. Ojemolon ${ }^{3}$, Hafeez Shaka ${ }^{1}$, Augustine Manadan ${ }^{4}$

1. Internal Medicine, John H. Stroger, Jr. Hospital of Cook County, Chicago, USA 2. Internal Medicine, University of Benin, Benin City, NGA 3. Anatomical Sciences, St. George's University, St. George, GRD 4. Rheumatology, John H. Stroger, Jr. Hospital of Cook County, Chicago, USA

Corresponding author: Ehizogie Edigin, ediginehizogie@yahoo.com

\section{Abstract \\ Objectives}

This study aimed to compare the outcomes of patients primarily admitted for atrial fibrillation (AF) with and without a secondary diagnosis of rheumatoid arthritis (RA). The primary outcome of interest was inpatient mortality. Hospital length of stay (LOS), total hospital charges, and odds of undergoing ablation and pharmacologic cardioversion were the secondary outcomes of interest.

\section{Methods}

Data were abstracted from the National Inpatient Sample (NIS) 2016 and 2017 databases. The NIS is the largest hospitalization database in the United States (US). The NIS was searched for hospitalizations for adult patients with $\mathrm{AF}$ as principal diagnosis with and without RA as secondary diagnosis using the International Classification of Diseases, 10th Revision (ICD-10) codes. Multivariate logistic and linear regression analysis was used accordingly to adjust for confounders.

\section{Results}

There were over 71 million discharges in the combined 2016 and 2017 NIS database. Out of 821,630 AF hospitalizations, 17,020 (2.1\%) had RA. Hospitalizations for AF with RA had 0.18 days' decrease in adjusted mean LOS $(\mathrm{p}=0.014$ ), and lower total hospital charges ( $\$ 38,432$ vs $\$ 39,175, \mathrm{p}=0.018$ ) compared to those without RA. AF hospitalizations with RA had similar inpatient mortality [1.1\% vs $0.91 \%$, adjusted odds ratio (AOR): $0.90,95 \%$ CI: $0.63-1.27, \mathrm{p}=0.540$ ] and odds of undergoing ablation (3.5\% vs $4.2 \%$, AOR: $1.1,95 \%$ CI: $0.87-1.30, \mathrm{p}=0.549)$ and pharmacologic cardioversion (0.38\% vs $0.38 \%$, AOR: $1.00,95 \%$ CI: 0.53-1.89, $\mathrm{p}=0.988$ ) compared to those without RA.

Received 08/11/2020 Review began 08/17/2020 Review ended 08/27/2020 Published 09/04/2020

๑) Copyright 2020

Edigin et al. This is an open access article distributed under the terms of the Creative Commons Attribution License CC-BY 4.0., which permits unrestricted use, distribution, and reproduction in any medium, provided the original author and source are credited.

\section{Conclusions}

Patients admitted for AF with coexisting RA were found to have lesser adjusted mean LOS and lower total hospital charges compared to those without RA. However, inpatient mortality and the odds of undergoing ablation and pharmacologic cardioversion were similar between both groups.

Categories: Cardiology, Internal Medicine, Rheumatology

Keywords: rheumatoid arthritis, atrial fibrillation, conduction disorders, pharmacologic cardioversion, ablation

\section{Introduction}

Atrial fibrillation (AF) is the most clinically relevant rhythm disorder; it is characterized by irregularly irregular rhythm and is a common cause of cardiovascular (CV) morbidity and mortality [1,2]. Rheumatoid arthritis (RA) is a chronic autoimmune disease that causes inflammatory arthritis and is characterized by synovial joint involvement as well as significant extra-articular manifestations and complications [3,4].

There is an established association between RA and CV diseases. CV mortality and morbidity are significantly higher in RA patients, with more than half of all RA-related deaths attributable to CV diseases $[4,5]$. RA has been shown to increase the incidence of AF with sustained inflammation as the major driver $[4,6]$. In a meta-analysis of three prospective studies, the pooled risk ratio for the development of AF in RA patients was found to be 1.29 (95\% CI: 1.05-1.59) compared to controls [4].

There is a scarcity of national population-based research on the outcomes of AF hospitalizations in RA patients compared to non-RA patients. We sought to bridge this knowledge gap. Our study sought to compare the outcomes of AF hospitalization in patients with RA to those without RA using national-level 
population data. Additionally, our study also investigated the likelihood of undergoing ablation or pharmacologic cardioversion in patients with AF with RA compared to those without RA. We used the data for the two most recent years from the National Inpatient Sample (NIS) database to address these clinically important issues.

\section{Materials And Methods \\ Data source}

We conducted a retrospective study of hospitalizations with a principal diagnosis of AF with and without a secondary diagnosis of RA from the NIS 2016 and 2017 databases. The NIS is maintained by the Agency for Healthcare Research and Quality. It is the largest available all-payer inpatient public database in the United States (US). It is designed as a stratified probability sample to be representative of all acute-care, nonfederal hospitals in the US. Hospitals are stratified according to bed size, teaching status, ownership, geographic region, and urban/rural status. A $20 \%$ probability sample of all hospitals within each stratum is then obtained. All hospitalizations from these hospitals are recorded and then weighted to ensure they remain representative nationally. Data from 47 statewide data organizations are included in the NIS 2016 and 2017 sampling frame. As much as 30 discharge diagnoses for each hospitalization can be recorded using the International Classification of Diseases, Tenth Revision (ICD-10) codes in NIS 2016, and 40 discharge diagnoses in the NIS 2017 database. Diagnoses are divided into principal and secondary diagnoses in the NIS database. The principal diagnosis is the main reason for the hospitalization. Secondary diagnoses are any diagnoses other than the principal diagnosis. Secondary diagnoses that preceded the index admission cannot be reliably distinguished from those that started during the index admission. All patient information in the NIS are de-personalized and available publicly, hence institutional board review and approval was not obtained for our study. The description of the NIS database is similar to a previously published NIS paper [7].

\section{Inclusion criteria and study variables}

The study population consisted of all inpatient hospitalizations recorded in the NIS 2016 and 2017. Race, gender, age, hospital characteristics, medical comorbidities, and primary and secondary outcomes were the study variables of interest. We used the following ICD-10 codes to identify principal/secondary diagnoses: AF - all 148.0, 148.1, 148.2, 148.91 codes; and RA - all M05 and M06 codes [see the supplementary table (Table 4) in the Appendix]. We studied baseline characteristics and outcomes for AF hospitalization with and without RA.

\section{Outcomes}

The primary outcome of interest was inpatient mortality. Hospital length of stay (LOS), total hospital charge, and odds of undergoing ablation and pharmacologic cardioversion were the secondary outcomes of interest. AF Hospitalizations with ablation were obtained by using ICD procedure codes (all 0258 codes) for "destruction of the conduction system of the heart", while those with pharmacologic cardioversion were obtained using ICD-10 procedure codes for "parenteral administration of antiarrhythmic agents".

\section{Statistical analysis}

Statistical analyses were performed using STATA, version 16 (StataCorp, College Station, TX). A univariate logistic regression analysis using all variables and comorbidities in Table 1 was used to calculate unadjusted odds ratios (ORs) for the primary outcome. All variables with p-values of $<0.1$ were included in a multivariate logistic regression model. Univariate association of variables and comorbidities with the primary outcome, highlighting the variables included in the multivariable logistic regression model, are displayed in Table 2 . $P$-values of $<0.05$ were considered significant in the multivariate analysis. Confounders were selected from the literature review. The Charlson Comorbidity Index was used to adjust for comorbidity burden. A multivariate logistic and linear regression model with all variables and comorbidities in Table 1 was used accordingly to adjust for confounders for the secondary outcomes.

\section{Results}

There were over 71 million discharges included in the combined 2016 and 2017 NIS database. Of those, 821,630 hospitalizations were adult patients, who had a principal ICD-10 code for AF; 17,020 (2.1\%) of these hospitalizations had RA as a secondary diagnosis, while 804,610 (97.9\%) hospitalizations did not have RA as a secondary diagnosis. Characteristics of AF hospitalizations with and without coexisting RA are displayed in Table 1 .

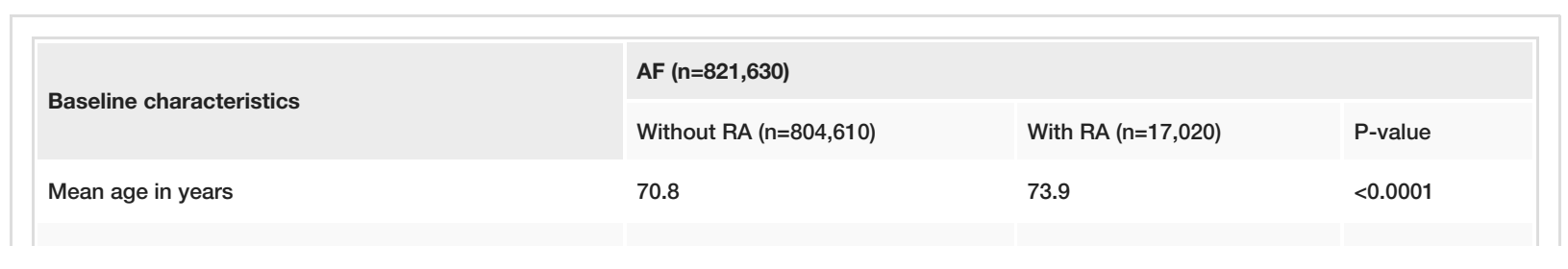




\section{Cureus}

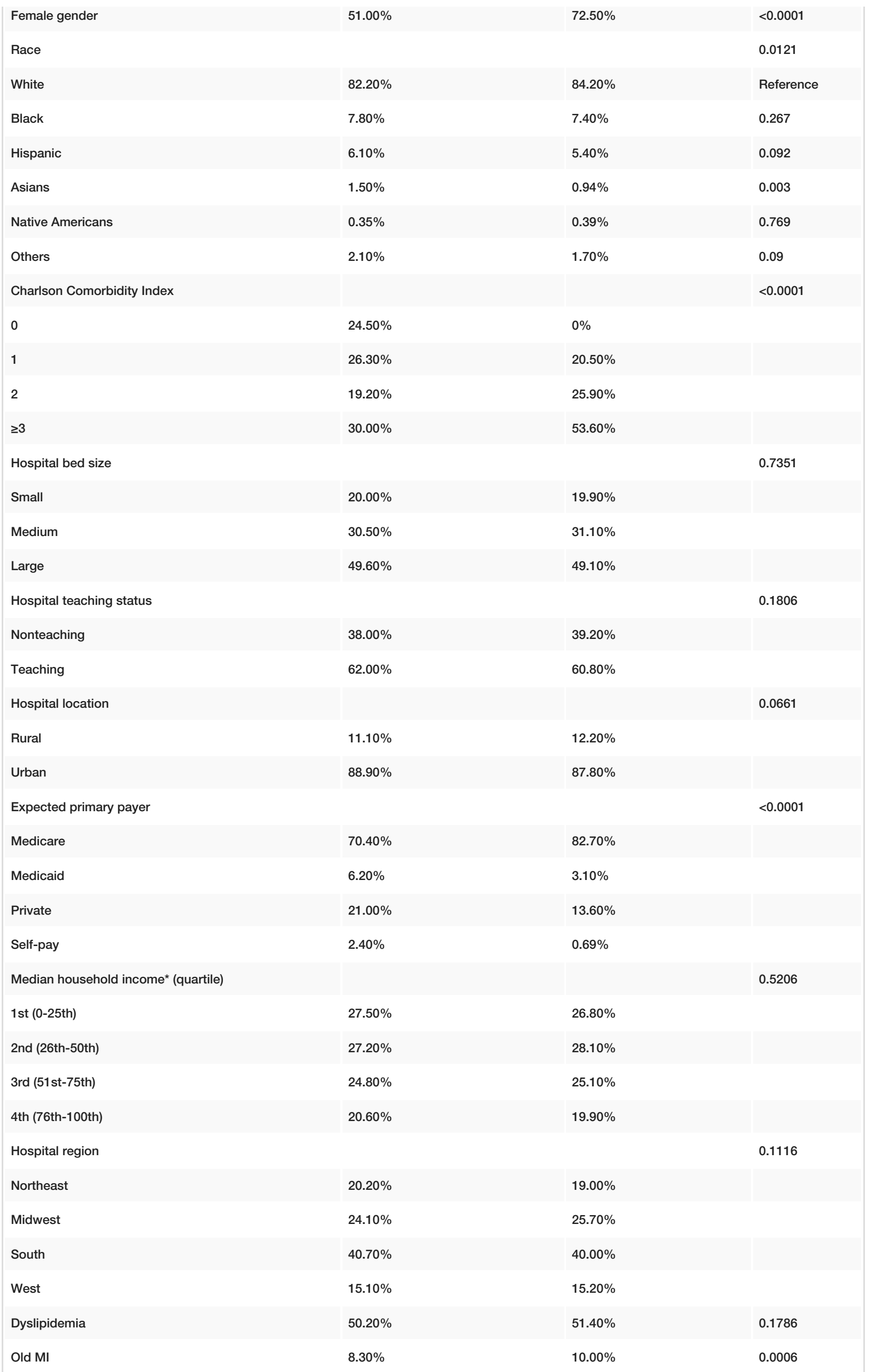




\section{Cureus}

\begin{tabular}{|c|c|c|c|}
\hline Old PCI & $1.10 \%$ & $0.97 \%$ & 0.4194 \\
\hline Old CABG & $7.20 \%$ & $7.00 \%$ & 0.7158 \\
\hline Old pacemaker & $5.40 \%$ & $5.80 \%$ & 0.3406 \\
\hline AICD & $3.00 \%$ & $2.00 \%$ & 0.0012 \\
\hline COPD & $19.40 \%$ & $26.70 \%$ & $<0.0001$ \\
\hline Carotid artery disease & $1.30 \%$ & $1.70 \%$ & 0.0821 \\
\hline Old stroke & $0.62 \%$ & $0.41 \%$ & 0.1268 \\
\hline Hypertension & $48.40 \%$ & $48.50 \%$ & 0.9559 \\
\hline Peripheral vessel disease & $4.20 \%$ & $5.10 \%$ & 0.0053 \\
\hline Hypothyroidism & $17.20 \%$ & $24.40 \%$ & $<0.0001$ \\
\hline DM type 1 and 2 & $28.00 \%$ & $24.60 \%$ & $<0.0001$ \\
\hline Obesity & $19.60 \%$ & $17.20 \%$ & 0.0004 \\
\hline CHF & $37.50 \%$ & $41.80 \%$ & $<0.0001$ \\
\hline CKD & $18.10 \%$ & $20.10 \%$ & 0.0026 \\
\hline Liver disease & $3.00 \%$ & $2.60 \%$ & 0.1694 \\
\hline Electrolyte derangement & $18.80 \%$ & $22.00 \%$ & $<0.0001$ \\
\hline Maintenance hemodialysis & $2.20 \%$ & $1.50 \%$ & 0.0098 \\
\hline $\mathrm{O}_{2}$ dependence & $3.40 \%$ & $5.00 \%$ & $<0.0001$ \\
\hline Smoking & $27.60 \%$ & $30.10 \%$ & 0.0012 \\
\hline Anemia & $15.70 \%$ & $23.20 \%$ & $<0.0001$ \\
\hline
\end{tabular}

\section{TABLE 1: Baseline characteristics of atrial fibrillation hospitalizations with and without rheumatoid arthritis}

*Median household income for the patient's Zip Code

AF: atrial fibrillation; RA: rheumatoid arthritis; MI: myocardial infarction; PCI: percutaneous coronary intervention; CABG: coronary artery bypass graft; COPD: chronic obstructive pulmonary disease; DM: diabetes mellitus; CHF: chronic congestive heart failure; CKD: chronic kidney disease; $\mathrm{O}_{2}$ : oxygen; AICD: presence of automated implantable cardioverter defibrillator

Patients in the RA group were older ( 73.9 vs 70.8 years, $\mathrm{p}<0.0001$ ) and the group had more females than males $(72.5 \%$ vs $51.0 \%, \mathrm{p}<0.0001)$. Also, the RA group had fewer cases of diabetes mellitus ( $24.6 \%$ vs $28 \%$, $\mathrm{p}<0.0001)$ and obesity $(17.2 \%$ vs $19.6 \%, \mathrm{p}=0.0004)$. In contrast, the RA group had more smokers ( $30.1 \%$ vs $27.6 \%, \mathrm{p}=0.0012)$ and more patients with peripheral vessel disease $(5.1 \%$ vs $4.2 \%, \mathrm{p}=0.0053)$ and old myocardial infarction ( $10 \%$ vs $8.3 \%$, $\mathrm{p}=0.0006)$. The univariate association of variables and comorbidities with the primary outcome are displayed in Table 2.

\begin{tabular}{|c|c|c|}
\hline Baseline variables & Odds ratio & P-value \\
\hline Age $^{\star}$ & 1.06 & $<0.0001$ \\
\hline Female gender ${ }^{\star}$ & 1.19 & $<0.0001$ \\
\hline \multicolumn{3}{|l|}{ Race $^{*}$} \\
\hline White & Reference & Reference \\
\hline Black & 1.17 & 0.085 \\
\hline Hispanic & 1.11 & 0.325 \\
\hline Asians & 1.19 & 0.421 \\
\hline
\end{tabular}




\section{Cureus}

\begin{tabular}{|c|c|c|}
\hline Native Americans & 0.99 & 0.979 \\
\hline Others & 0.55 & 0.015 \\
\hline Charlson Comorbidity Index* & 1.39 & $<0.0001$ \\
\hline \multicolumn{3}{|l|}{ Hospital bed size } \\
\hline Small & Reference & Reference \\
\hline Medium & 0.98 & 0.734 \\
\hline Large & 0.91 & 0.167 \\
\hline Hospital teaching status & 1.02 & 0.755 \\
\hline Hospital location & 0.99 & 0.979 \\
\hline \multicolumn{3}{|l|}{ Expected primary payer* } \\
\hline Medicare & Reference & Reference \\
\hline Medicaid & 0.58 & $<0.0001$ \\
\hline Private & 0.35 & $<0.0001$ \\
\hline Self-pay & 0.45 & 0.001 \\
\hline \multicolumn{3}{|c|}{ Median household income ${ }^{\star \star}$ (quartile) $^{\star}$} \\
\hline 1st (0-25th) & Reference & Reference \\
\hline 2nd (26th-50th) & 0.93 & 0.293 \\
\hline 3rd (51st-75th) & 0.83 & 0.012 \\
\hline 4th (76th-100th) & 0.74 & $<0.0001$ \\
\hline \multicolumn{3}{|l|}{ Hospital region* } \\
\hline Northeast & Reference & Reference \\
\hline Midwest & 0.9 & 0.188 \\
\hline South & 0.99 & 0.836 \\
\hline West & 1.16 & 0.086 \\
\hline Dyslipidemia* & 0.64 & $<0.0001$ \\
\hline Old MI & 0.88 & 0.228 \\
\hline Old PCI & 0.83 & 0.49 \\
\hline Old CABG* & 1.18 & 0.066 \\
\hline Old pacemaker & 0.91 & 0.44 \\
\hline AICD & 1.01 & 0.926 \\
\hline COPD $^{*}$ & 2.05 & $<0.0001$ \\
\hline Carotid artery disease & 1.13 & 0.605 \\
\hline Old stroke ${ }^{\star}$ & 7.61 & $<0.0001$ \\
\hline Hypertension* & 0.45 & $<0.0001$ \\
\hline Peripheral vessel disease ${ }^{*}$ & 1.6 & $<0.0001$ \\
\hline Hypothyroidism & 1.01 & 0.912 \\
\hline DM type 1 and 2 & 1.03 & 0.583 \\
\hline Obesity* & 0.49 & $<0.0001$ \\
\hline $\mathrm{CHF}^{\star}$ & 2.56 & $<0.0001$ \\
\hline
\end{tabular}




\section{Cureus}

\begin{tabular}{|lcc|} 
CKD $^{*}$ & 2.43 & $<0.0001$ \\
Liver disease $^{*}$ & 4.93 & $<0.0001$ \\
Electrolyte derangement $^{*}$ & 4.82 & $<0.0001$ \\
Maintenance hemodialysis $^{*}$ & 2.12 & $<0.0001$ \\
$\mathrm{O}_{2}$ dependence $^{\star}$ & 2.43 & $<0.0001$ \\
Smoking $^{*}$ & 0.82 & 0.002 \\
Anemia $^{*}$ & 2.66 & $<0.0001$ \\
\hline
\end{tabular}

\section{TABLE 2: Univariate association of baseline variables with inpatient mortality}

*Variable included in the multivariable logistic regression model; **Median household income for the patient's Zip Code

MI: myocardial infarction; PCI: percutaneous coronary intervention; CABG: coronary artery bypass graft; COPD: chronic obstructive pulmonary disease; DM: diabetes mellitus; AICD: presence of automated implantable cardioverter defibrillator; CHF: chronic congestive heart failure; CKD: chronic kidney disease; $\mathrm{O}_{2}$ : oxygen

Of note, 7,520 adult AF hospitalization (0.9\%) resulted in inpatient mortality; 190 (1.1\%) of the deaths occurred amomg patients with coexisting RA vs 7,330 (0.9\%) for those without coexisting RA ( $\mathrm{p}=0.2121)$. Hospitalizations for AF with RA had 0.18 days' decrease in adjusted mean LOS ( $\mathrm{p}=0.014$ ), and lower total hospital charge ( $\$ 38,432$ vs $\$ 39,175, \mathrm{p}=0.018$ ) compared to those without RA. AF hospitalizations with RA had similar inpatient mortality [1.1\% vs $0.91 \%$, adjusted odds ratio (AOR): $0.90,95 \%$ CI: $0.63-1.27, \mathrm{p}=0.540$ ] and odds of undergoing ablation (3.5\% vs $4.2 \%$, AOR: 1.1, 95\% CI: $0.87-1.30, \mathrm{p}=0.549)$ and pharmacologic cardioversion ( $0.38 \%$ vs $0.38 \%$, AOR: $1.00,95 \%$ CI: $0.53-1.89, \mathrm{p}=0.988$ ) compared to those without RA (Table 3).

\begin{tabular}{|c|c|c|c|c|}
\hline \multirow{2}{*}{ Outcomes } & AF with RA $(n=17,020)$ & AF without RA $(n=804,610)$ & Adjusted odds ratio & P-value \\
\hline & $\%$ & $\%$ & $(95 \% \mathrm{Cl})$ & \\
\hline \multicolumn{5}{|l|}{ Primary outcome } \\
\hline In-hospital mortality & 1.1 & 0.91 & $0.90(0.63-1.27)$ & 0.540 \\
\hline \multicolumn{5}{|l|}{ Secondary outcomes } \\
\hline Ablation & 3.5 & 4.2 & $1.1(0.87-1.30)$ & 0.549 \\
\hline \multirow[t]{2}{*}{ Pharmacologic cardioversion } & 0.38 & 0.38 & $1.00(0.53-1.89)$ & 0.988 \\
\hline & & & Adjusted mean difference & \\
\hline LOS, mean, days & 3.7 & 3.4 & $-0.18(-\{0.33-0.04\})$ & $0.014^{\star}$ \\
\hline Iotal charge, mean, USD & 38,432 & 39,175 & $-2,035(-\{3,725-346\})$ & $0.018^{\star}$ \\
\hline
\end{tabular}

\section{TABLE 3: Clinical outcomes of atrial fibrillation hospitalizations with and without rheumatoid} arthritis

*Statistically significant

LOS: length of stay at hospital; Cl: confidence interval; AF: atrial fibrillation; RA: rheumatoid arthritis; USD: United States Dollar

\section{Discussion}

The major findings of this study were as follows: 1) no difference in mortality between AF hospitalizations with and without RA; 2) patients hospitalized for AF with RA had lesser adjusted LOS and total hospital charges compared to those without RA; and 3) AF hospitalizations with and without RA had similar rates of interventions such as ablation and pharmacologic cardioversion.

RA is the most common inflammatory joint disease with a significant CV disease burden [2]. The most 
common arrhythmia among RA patients is AF, with a higher incidence rate compared to the general population $[2,8]$. The underlying mechanism of increased risk of AF in patients with RA has been extensively studied and several explanations have been offered. Most studies point to the chronic inflammatory state with significant inflammatory marker levels playing an important role in the development of AF indirectly by accelerating the rate of ischemic heart disease and congestive heart failure and directly on the cardiac conduction system $[9,5]$. The direct effect proposed in another study on the conduction system includes the effect of elevated circulatory antibodies to the conducting system, which leads to increased P-wave dispersion and increase in left atrium (LA) size, which is a known predictor of AF [8]. A more recent study in 2020 by Milton Parker showed that increased epicardial adipose expansion and inflammation found in RA led to coronary microvascular dysfunction and fibrotic changes in the LA leading to myopathy, increased LA stiffness, and blood stasis, resulting in AF [10].

Traditional risk factors for CV and cerebrovascular diseases are generally more prevalent in RA patients [11]. The RA group in our study had fewer statistically significant traditional CV risk factors such as DM and obesity. However, the RA group had a statistically significant higher number of smokers and more patients with peripheral vascular disease, old myocardial infarction, chronic kidney disease, and congestive heart failure. RA also tends to alter the lipid profile, characterized by low high-density lipoprotein (HDL), and high pro-atherogenic lipoprotein-a (Lpa) levels [12,13]. Our study did not find any statistically significant difference in dyslipidemia between the RA and non-RA groups.

A significant number of studies have clearly shown the increased incidence risk of AF in RA patients. A cohort study using the Danish National Registry showed a $40 \%$ increased incidence rate in patients with RA compared to the general population [9]. In a meta-analysis, the pooled risk ratio for AF development was found to be 1.29, showing an increased risk for developing AF in RA patients [4].

However, very few studies have been conducted on the outcomes of AF in RA patients. A similar population-based cohort study in Olmsted County, Minnesota showed no difference in mortality between AF patients with and without RA [14]. The study was limited because it involved a small subset of the US population with only 1,626 patients. Our study used a nationwide US hospitalization database and it showed similar results to the study in Minnesota. Our study concluded that mortality outcome is not influenced by the presence of RA. More studies are needed to explore the mechanism behind our findings.

This study has several strengths. Firstly, it utilized information from the NIS, a large nationwide dataset, to provide a large sample size, thereby greatly increasing the power of the study. Secondly, the nature of the database allowed us to compare the baseline demographic characteristics and various hospital outcomes between AF hospitalizations with and without concomitant RA.

This study has some limitations. Firstly, NIS database studies are subject to all the biases associated with retrospective studies. Secondly, the NIS uses ICD-10 codes to characterize diagnoses and hospitalization events, and hence there is a possibility of errors associated with coding. Thirdly, most of the ICD-10 billing codes used by the database fail to grade disease severity. Thus, we could not determine if underlying disease severity affected the outcome of AF patients with RA. Fourthly, this report reflects data on AF hospitalizations rather than on individual patients, and, therefore, individuals hospitalized multiple times with the same principal diagnosis would be counted multiple times. Finally, data on medications used during hospitalization, adherence to immunosuppressants such as disease-modifying anti-rheumatic drugs (DMARDs) and biologic agents, and laboratory results, which could indicate underlying disease severity and inflammatory activity, are not available in the NIS database.

\section{Conclusions}

Based on our findings, patients admitted primarily for AF with a secondary diagnosis of RA had lesser adjusted mean LOS and total hospital charges compared to those without RA. However, inpatient mortality and the likelihood of undergoing ablation and pharmacologic cardioversion were similar between both groups.

\section{Appendices}




\section{Cureus}

\section{ICD-10 codes}

Diagnosis codes

AF

RA

Procedure codes

Ablation

Pharmacologic cardioversion

Comorbidities

Dyslipidemia

Old MI

Old PCl

Old CABG

Old pacemaker

AICD

Chronic obstructive pulmonary disease

Carotid artery disease

Old stroke

Hypertension

Peripheral vascular disease

Hypothyroidism

Diabetes mellitus type 1 and 2

Obesity

Congestive heart failure

Chronic kidney disease

Liver disease

Electrolyte derangement

Maintenance dialysis

$\mathrm{O}_{2}$ dependence

Smoking

Anemia

\section{$148.0,148.1,148.2,148.91$}

M05, M06

258

3E030RZ, 3E033RZ, 3E040RZ, 3E043RZ, 3E050RZ, 3E053RZ, 3E060RZ, 3E063RZ

E78

1252

Z9861

Z951

Z950

Z95810

$\mathrm{J} 41, \mathrm{~J} 42, \mathrm{~J} 43, \mathrm{~J} 44$

1652

163

110

1739

E03

E10, E11

E660, E6601, E6609, E661, E662, E668, E669

150

N18

K70, K71, K72, K73, K74, K75, K76, K77

E870, E871, E872, E873, E874, E875, E876

Z992

Z9981

Z87891, F17200

D50, D51, D52, D53, D55, D56, D57, D58, D59, D60, D61, D62, D63, D64

\section{TABLE 4: Supplementary table containing used ICD-10 codes}

AF: atrial fibrillation; RA: rheumatoid arthritis; MI: myocardial infarction; PCI: percutaneous coronary intervention; CABG: coronary artery bypass graft; COPD: chronic obstructive pulmonary disease; DM: diabetes mellitus; $\mathrm{CHF}$ : chronic congestive heart failure; CKD: chronic kidney disease; $\mathrm{O}_{2}$ : oxygen; AICD: presence of automated implantable cardioverter defibrillator; ICD-10: International Classification of Diseases, Tenth Revision

\section{Additional Information}

\section{Disclosures}

Human subjects: Consent was obtained by all participants in this study. N/A issued approval N/A. All patient information in NIS are de-personalized and available publicly, hence institutional board review and approval was not obtained. Animal subjects: All authors have confirmed that this study did not involve 
animal subjects or tissue. Conflicts of interest: In compliance with the ICMJE uniform disclosure form, all authors declare the following: Payment/services info: All authors have declared that no financial support was received from any organization for the submitted work. Financial relationships: All authors have declared that they have no financial relationships at present or within the previous three years with any organizations that might have an interest in the submitted work. Other relationships: All authors have declared that there are no other relationships or activities that could appear to have influenced the submitted work.

\section{References}

1. Goudis CA: Chronic obstructive pulmonary disease and atrial fibrillation: an unknown relationship . J Cardiol. 2017, 69:699-705. 10.1016/j.jjcc.2016.12.013

2. Echouffo-Tcheugui JB, Shrader P, Thomas L, et al.: Care patterns and outcomes in atrial fibrillation patients with and without diabetes; ORBIT-AF registry. J Am Coll Cardiol. 2017, 70:1325-35. 10.1016/j.jacc.2017.07.755

3. Elbadawi A, Ahmed HMA, Elgendy IY, et al.: Outcomes of acute myocardial infarction in patients with rheumatoid arthritis (Epub ahead of print). Am J Med. 2020, 10.1016/j.amjmed.2020.02.039

4. Ungprasert P, Srivali N, Kittanamongkolchai W: Risk of incident atrial fibrillation in patients with rheumatoid arthritis: a systematic review and meta-analysis. Int J Rheum Dis. 2017, 20:434-41. 10.1111/1756-185X.12820

5. Lazzerini PE, Capecchi PL, Laghi-Pasini F: Systemic inflammation and arrhythmic risk: lessons from rheumatoid arthritis. Eur Heart J. 2017, 38:1717-27. 10.1093/eurheartj/ehw208

6. Lazzerini PE, Capecchi PL, Acampa M, Galeazzi M, Laghi-Pasini F: Arrhythmic risk in rheumatoid arthritis: the driving role of systemic inflammation. Autoimmun Rev. 2014, 13:936-44. 10.1016/j.autrev.2014.05.007

7. Edigin E, Eseaton P, Kaul S, et al.: Systemic sclerosis is not associated with worse outcomes of patients admitted for ischemic stroke: analysis of the National Inpatient Sample. Cureus. 2020, 12:e9155. 10.7759/cureus. 9155

8. Gawałko M, Balsam P, Lodziński P, Grabowski M, Krzowski B, Opolski G, Kosiuk J: Cardiac arrhythmias in autoimmune diseases. Circ J. 2020, 84:685-94. 10.1253/circj.CJ-19-0705

9. Lindhardsen J, Ahlehoff $\mathrm{O}$, Gislason $\mathrm{GH}$, et al.: Risk of atrial fibrillation and stroke in rheumatoid arthritis: Danish nationwide cohort study. BMJ. 2012, 344:e1257. 10.1136/bmj.e1257

10. Packer M: Characterization, pathogenesis, and clinical implications of inflammation-related atrial myopathy as an important cause of atrial fibrillation. J Am Heart Assoc. 2020, 9:e015343. 10.1161/JAHA.119.015343

11. Jagpal A, Navarro-Millán I: Cardiovascular co-morbidity in patients with rheumatoid arthritis: a narrative review of risk factors, cardiovascular risk assessment and treatment. BMC Rheumatol. 2018, 2:10. 10.1186/s41927-018-0014-y

12. Choy E, Sattar N: Interpreting lipid levels in the context of high-grade inflammatory states with a focus on rheumatoid arthritis: a challenge to conventional cardiovascular risk actions. Ann Rheum Dis. 2009, 68:4609. 10.1136/ard.2008.101964

13. Full LE, Monaco C: Targeting inflammation as a therapeutic strategy in accelerated atherosclerosis in rheumatoid arthritis. Cardiovasc Ther. 2011, 29:231-42. 10.1111/j.1755-5922.2010.00159.x

14. Bacani AK, Crowson CS, Roger VL, Gabriel SE, Matteson EL: Increased incidence of atrial fibrillation in patients with rheumatoid arthritis. Biomed Res Int. 2015, 2015:809514. 10.1155/2015/809514 